\title{
Abdominal tuberculosis presenting as mass per abdomen: a case report
}

\author{
Balaji ${ }^{1}$, Divya $\mathbf{N}^{2}$ \\ ${ }^{1}$ Dr Balaji, Professor, ${ }^{2}$ Dr Divya Narayanan Kutty, Postgraduate, Department of Pediatrics, Adichunchanagiri Institute of \\ Medical Sciences, B.G. Nagara, Nagamangala Taluk, Mandya District, Karnataka, India.
}

Address for Correspondence: Dr Divya Narayanan Kutty, E-mail: dichu5985@gmail.com

\begin{abstract}
Tuberculosis (TB) can involve any part of the gastrointestinal tract, the peritoneum, lymphnodes and the pancreatobiliary system. The primary site of TB is usually lung, from where it disseminates to other parts of the body. TB of the gastrointestinal tract is the sixth most frequent form of extrapulmonary tuberculosis. The incidence of abdominal tuberculosis in children in India is estimated to be 2.2 million as in the year 2014 and India has the world's largest tuberculosis cases of around $26 \%$. It can have a varied presentation and can mimic any abdominal pathology. A high index of suspicion is an important factor in early diagnosis. Here we present a case of abdominal tuberculosis who presented as mass per abdomen.
\end{abstract}

Keywords: Tuberculosis, Extrapulmonary, Abdominal tuberculosis

\section{Introduction}

Tuberculosis (TB) is a life threatening disease which can virtually affect any part of the gastrointestinal tract [1]. According to World Health Organization report 2015, there was an estimated annual incidence of 9 million of TB globally [2]. India has the world's largest tuberculosis cases which is around $26 \%$ of the world TB cases, followed by China and South Africa [1].

The primary site of TB is usually lung, from where it disseminates to other parts of the body. TB of the gastrointestinal tract is the sixth most frequent form of extra-pulmonary site, after lymphatic, genitourinary, bone and joint, miliary and meningeal tuberculosis [3].
The abdominal TB is usually diagnosed late due to its rare presentation and the diagnosis requires a high index of suspicion [4]. It affects both genders equally and the most common age of presentation is $10-25$ yrs [5].

The abdominal TB usually occurs in four forms: tuberculous lymphadenopathy, peritoneal tuberculosis, gastro-intestinal (GI) tuberculosis and visceral tuberculosis. Computed tomography (CT) appears to be the imaging modality of choice in the detection and assessment of abdominal TB [1]. It can present as either mass per abdomen or peritonitis thinking of other common surgical conditions.

\section{Case Report}

A 17 year old adolescent boy presented with a history of weight loss since 3 months and diffuse abdominal pain since 1 month. He was previously admitted 1 month back for similar complaints and was treated medically in view of appendicitis. There was a history of recurrence of the symptoms. Physical examination revealed a protuberant abdomen. On palpation there was a mass with undefined borders of a doughy feel with associated hepatomegaly and a generalised tenderness. His blood investigations and urine examination were normal except for $\mathrm{Hb} 9 \mathrm{gm} \%$ and ESR 34.

The plain abdominal radiographs were nonspecific. Ultrasonography of the abdomen showed mild hepatomegaly with cavernous formation of portal vein and mild echogenic ascites with enlarged necrotic peripancreatic nodes and mesenteric nodes. Child was further evaluated with CT abdomen that showed mild ascites, small bowel matted together in central abdomen and enlarged mesenteric lymph nodes.

Manuscript received: $5^{\text {th }}$ January 2017

Reviewed: $11^{\text {th }}$ January 2017

Author Corrected: $20^{\text {th }}$ January 2017

Accepted for Publication: $28^{\text {th }}$ January 2017 


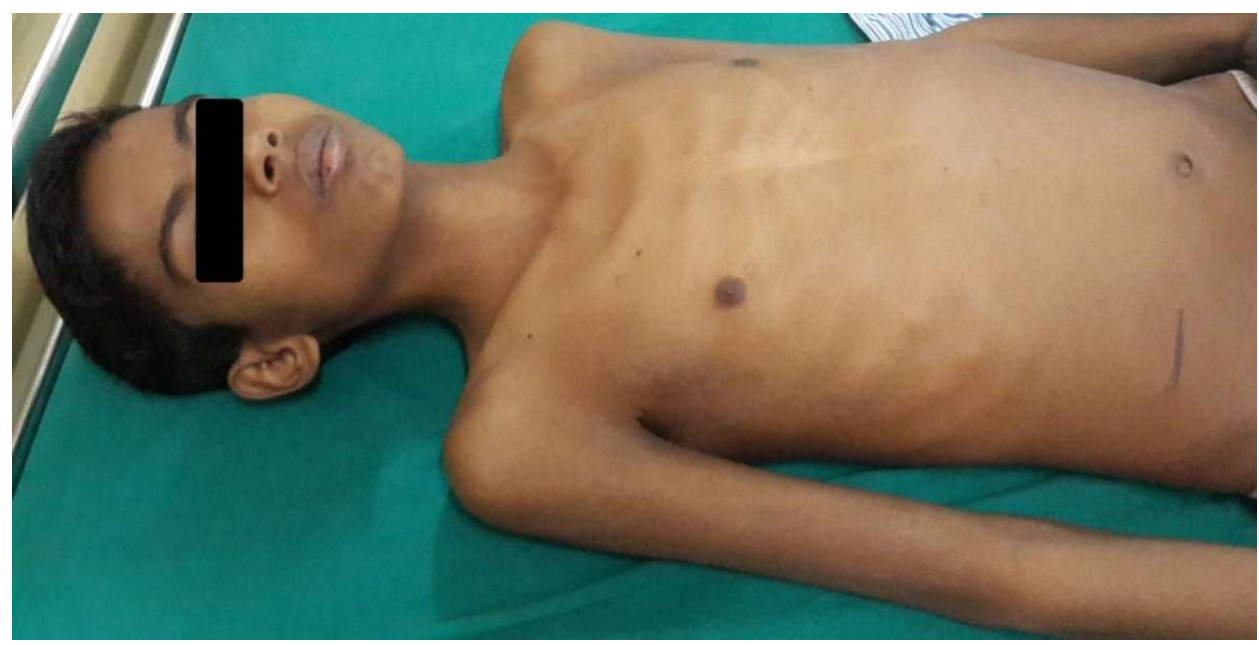

Figure-1: Child with the mass

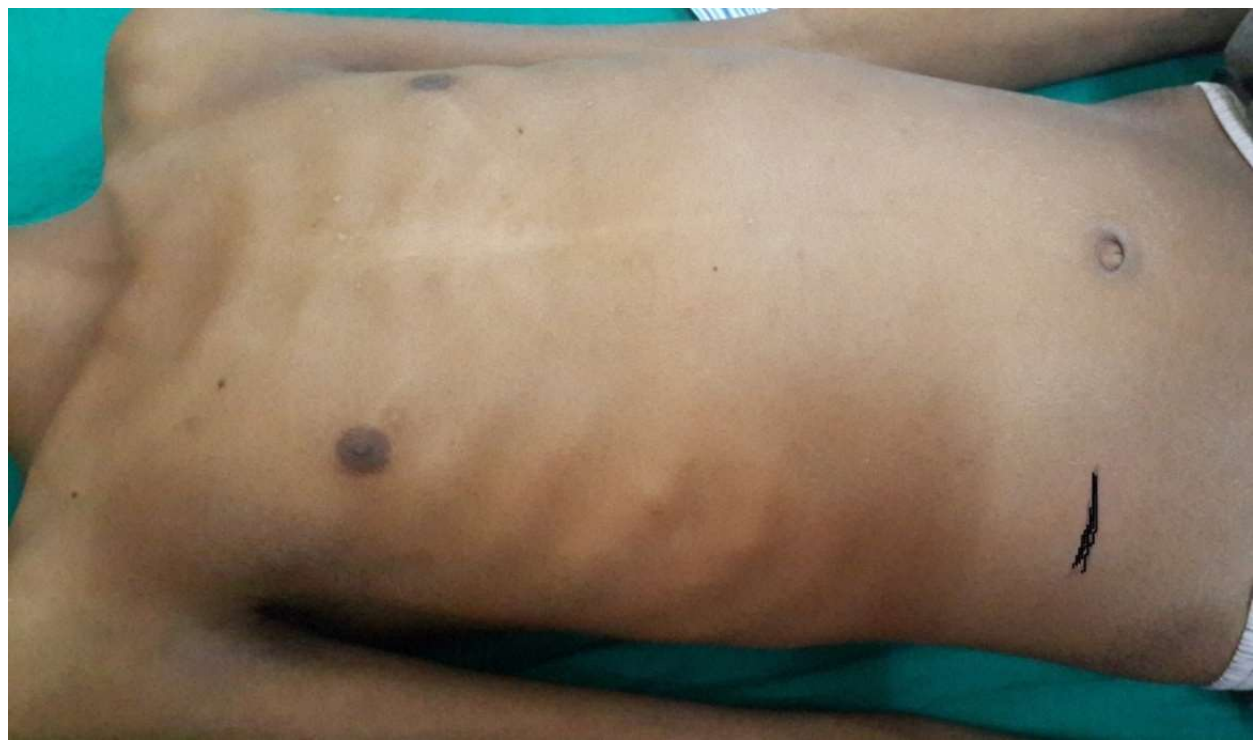

Figure-2: Mass with undefined borders of doughy feel

A differential diagnosis of abdominal tuberculosis, malignancy or an inflammatory disease was made. Ascitic fluid tapping was done and the fluid was turbid in nature. Mantoux test and sputum for AFB were negative. Ascitic fluid cytology suggested 700cells/mm3 with moderately cellular smear comprising of abundant lymphocytes (90\%) and few neutrophils $(10 \%)$ with a good number of reactive mesothelial cells. Ascitic fluid protein was elevated (3.8) and LDH was 650. The ascitic fluid ADA levels were 37U/L. (>36U/L is suggestive of tuberculosis)

A final diagnosis of abdominal tuberculosis was made. He was started on ATT (RNTCP: CAT-1) and gradually his symptoms improved. He did not have any complications during the follow ups.

\section{Discussion}

Abdominal tuberculosis constitutes $11-16 \%$ of the extrapulmonary TB. India is the country with the highest burden of TB. The World Health Organisation (WHO) statistics for 2014 gives an estimated incidence of 2.2 million cases of TB for India out of a global incidence of 9 million. The estimated TB prevalence for
2014 is 2.5 million [2]. It is estimated that about $40 \%$ of the Indian population is infected with TB bacteria, the vast majority of whom have latent rather than active TB. Abdominal tuberculosis develops with the reactivation of a quiet focus. The disease develops by lymphohematogenous spread from the pulmonary focus 
or by swallowing the bacilli. The abdominal TB usually occurs in four forms: tuberculous lymphadenopathy which is the most common type, peritoneal tuberculosis, gastrointestinal (GI) tuberculosis and visceral tuberculosis involving the solid organs $[6,7]$. The most common involvement is intestinal (42\%), in which ileocaecal region and small bowel and colon are involved $[8,9]$.

Abdominal tuberculosis can present with various clinical presentations and the diagnosis is based on the radiological and histopathological evidence. In our case the child presented with a mass per abdomen. Examination revealed a mass with a doughy feel gave a suspicion of abdominal tuberculosis or a malignancy. Radiologically, the presence of free fluid, small bowel adhesions and mesenteric lymphadenopathy suggested a peritoneal involvement.

Ascitic fluid analysis clinched the diagnosis. The tubercular ascitic fluid has protein more than $3 \mathrm{~g} / \mathrm{dL}$, with a total cell count of $150-4000 / \mu \mathrm{L}$ and consists predominantly of lymphocytes [10]. The ascitic fluid to blood glucose ratio is less than 0.96 and serum ascitic albumin gradient is less than $1.1 \mathrm{~g} / \mathrm{dL}$ [11]. Ascitic fluid adenosine deaminase (ADA) levels are elevated in tubercular ascites. Serum ADA level above $54 \mathrm{U} / \mathrm{L}$, ascitic fluid ADA level above $36 \mathrm{U} / \mathrm{L}$ and an ascitic fluid to serum ADA ratio more than 0.98 are suggestive of tuberculosis [12].

Showing the tubercular bacilli in culture or demonstrating the alcohol-acid-resistant bacilli or caseous granuloma histologically is diagnostic. However, demonstrating the bacilli is possible only in $20-70 \%$ of the cases. A negative intradermal reaction does not exclude the diagnosis. The Mantoux test has been found to be positive in $50-78 \%$ of patients with abdominal tuberculosis [13].

Various molecular and immunological modalities have been used in the rapid diagnosis of abdominal TB. PCR, Real-time assay and Multiplex PCR. Multiplex PCR has sensitivity and specificity of $90 \%$ and $100 \%$, respectively in confirmed (AFB/culture/histopathology) cases of gastrointestinal TB and positive results in $72.41 \%$ of the suspected gastrointestinal TB cases [14].

Our patient finally improved after starting antitubercular treatment. All the diagnosed cases of gastrointestinal TB should receive at least 6 mo of antituberculous therapy which includes initial two months of therapy with isoniazid, rifampicin, pyrazinamide and ethambutol thrice weekly [10].

\section{Conclusion}

Abdominal TB can affect any part of the GI tract. It usually involves the ileocaecal region. The symptoms can be non specific. The radiological and histopathological evidence aids in the diagnosis. A high index of suspicion helps in the diagnosis. Various immunological modalities help in the rapid diagnosis. The treatment is usually medical with antitubercular drugs and surgery is done unless absolutely indicated.

Funding: Nil, Conflict of interest: None initiated, Perission from IRB: Yes

\section{References}

1. Debi U, Ravisankar V, Prasad KK, Sinha SK, Sharma AK. Abdominal tuberculosis of the gastrointestinal tract: revisited. World J Gastroenterol. 2014 Oct 28;20(40):14831-40. doi: 10.3748/wjg.v20. i40.14831.

2. World Health Organization. Global tuberculosis report 2015. Geneva: WHO. 23 Oct; 2015.

3. Paustian FF. Tuberculosis of the intestine. In: Bockus HL, editor. Gastroenterology, vol.11, 2nd ed. Philadelphia : W.B. Saunders Co.; 1964 p. 311.

4. Mukewar S, Mukewar S, Ravi R, Prasad A, S Dua K. Colon tuberculosis: endoscopic features and prospective endoscopic follow-up after anti-tuberculosis treatment. Clin Transl Gastroenterol. 2012 Oct 11;3:e24. doi: 10. 1038/ctg.2012.19.

5. Sanai FM, Bzeizi KI. Systematic review: tuberculous peritonitis--presenting features, diagnostic strategies and treatment. Aliment Pharmacol Ther. 2005 Oct 15; 22 (8):685-700.

6. de Jesus LE, Marques AM, Rocha MS, Brom BR, Siqueira RR. Left colon stenosis caused by tuberculosis. J Pediatr Surg. 2004 Oct;39(10):e5-7.

7. Bhansali SK. Abdominal tuberculosis. Experiences with 300 cases. Am J Gastroenterol. 1977 Apr;67 (4):324-37.

8. Khan R, Abid S, Jafri W, Abbas Z, Hameed K, Ahmad Z. Diagnostic dilemma of abdominal tuberculosis in nonHIV patients: an ongoing challenge for physicians. World J Gastroenterol. 2006 Oct 21; 12(39):6371-5. 
9. Aston NO. Abdominal tuberculosis. World J Surg. 1997 Jun;21(5):492-9.

10. Sharma MP, Bhatia V. Abdominal tuberculosis. Indian J Med Res. 2004 Oct;120(4):305-15.

11. Wilkins EG. Tuberculosis peritonitis: diagnostic value of the ascitic/blood glucose ratio. Tubercle. 1984 Mar;65(1):47-52.

12. Bhargava DK, Gupta M, Nijhawan S, Dasarathy S, Kushwaha AK. Adenosine deaminase (ADA) in peritoneal tuberculosis: diagnostic value in ascitic fluid and serum. Tubercle. 1990 Jun;71(2):121-6.

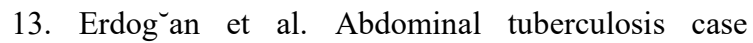
mimicking abdominal mass. Annals of Pediatric Surgery 2013;9:81-3.

14. Sharma K, Sinha SK, Sharma A, Nada R, Prasad KK, Goyal K, Rana SS, Bhasin DK, Sharma M. Multiplex PCR for rapid diagnosis of gastrointestinal tuberculosis. J Glob Infect Dis. 2013 Apr;5(2):49-53. doi: 10. 4103/0974-777X.112272.

\section{How to cite this article?}

Balaji, Divya N. Abdominal tuberculosis presenting as mass per abdomen: a case report. J PediatrRes.2017;4(01):3841.doi:10.17511/ijpr.2017.i01.08 